Restons-en là, et concluons à notre tour :

$\mathbf{I}^{\circ}$ Que l'on peut avoir parfaitement le droit d'être sceptique sur le rôle néfaste des Libellules vis-à-vis des Poissons. S'il y a des exceptions, ce ne sont que des exceptions qui confirment la règle ;

$2^{\circ}$ Que l'on a raison de noter ces organismes, et avec soin, dans le relevé des composantes sitétiques d'un biotope ichthyen ;

$3^{\circ} \mathrm{Qu}$ 'il convient donc de savoir ce que sont les Libellules pour propager celles qui conviennent et là où il convient ;

$4^{\circ}$ Que cet aménagement ne se pourra faire qu'à la condition que le dit biotope offre à ces larves de quoi se nourrir pour éviter le développement de leurs instincts carnassiers aux dépens du menu peuple poissonnier ( $I$ ).

(A suivre).

\title{
RÉFLEXIONS \\ SUR LA PRODUGTION ET LA VENTE DU POISSON D'ÉTANG
}

Par le Comte de NEUFBOURG

Président du Syndicat des étangs du Forez.

I. - Les Allemands ont raison de livrer la Carpe en automne et la Tanche en hiver. D'abord la Carpe supporte mieux le transport pendant la saison incertaine, coupée de temps lourds. Ensuite la Tanche fait moins de déchet en viviers et s'y conserve mieux, jusqu'au Carệme. Enfin, si l'on gave de Tanches le consommateur dès le début, jl est lassé avant l'écoulement des Carpes, d'autant que la ménagère préfère la Tanche, plus petite et plus vite préparée.

La sagesse serait, dans les Syndicats de producteurs, de s'engager à ne livrer jamais plus de $20 \%$ de Tanches aux marchands avant Noël. Il faut des viviers ? Nous répétons depuis $\mathrm{r} 5$ ans que tout étang dépourvu de vivier est de demi-valeur.

En fait, le marchand demande moitié Tanche, et si le producteur, ce qui est le cas habituel, n'en a que ro ou ri \%, il paye très bon marché le tout ou laisse de la Carpe pour plus tard ; ce qui est le contraire du procédé avantageux à l'écoulement des pêches.

\footnotetext{
1) lillustration de ret article. comme celle des prócédents. est extraite du Manuei des Pêcheurs, Pisciculteurs et Gardes-Peche, edité par la Sociélés Centrale pour la protection de la pêche fluviale. Prix : 5 belgas, à envoyer à M. Lestage, 66, avenue de la Floride, Uccle, Belgique.
} 
L'aire des Syndicats est trop étendue : il faudrait une section syndicale par diamètre de 10 ou 12 lieues, avec un vivier central où serait, pour les syndiqués dépourvus de dépôts, reçue la Tanche d'automne seulement, pour être vendue après Noël. Ainsi le Commerce serait obligé d'écouler une grande partie de la Carpe avant Noël. Il ne serait cédé de Tanche, après Noël, qu'aux marchands justifiant avoir acheté tant ou tant de Carpes en automne.

Cet avantage du vivier central obligerait les exploitants à se syndiquer. De plus, les syndiqués s'engageraient d̀ ne jamais vendre d'empoissonnage aux non-syndiqués, gâcheurs et profiteurs égoistes des efforts de leurs voìsins. Ceci, ce serait enfin du syndicalisme, de l'esprit de corps.

Toutes les objections, dont le Français est toujours abondamment pourvu contre toute initiative, sont spécieuses. La Tanche serait prise saine ou véreuse ; de moins de 100 grammes, de 100 à 200 , de plus de 200 grammes; etc. Il y a un effort à fournir ? Oui. Veut-on améliorer le marché ou rester la proie des fabricants du décordre ?

II. - La faible consommation française de poisson d'eau douce vient surtout de la mauvaise Carpe produite. En Forez, la consommation a quadruplé depuis que fonctionne la garantie syndicale.

La production reste mauvaise parce que le Commerce achète d'abord le poisson des producteurs négligents, dépourvus de viviers, ignorants des cours, des procédés d'élevage et de vente. Ces péchès s'achètent moitié prix, et si, malgré cela, le marchand ne gagne rien sur la mauvạise Carpe, il se rattrape sur la Tanche et le Brochet qui ne lui ont pas coûté plus cher. Mon ami du Pré-Tevciard, qui empoissonne avec son déchet, trouve toujours beaucoup de Broehets, qu'il lui faut vendre sur-le-champ à son fidèle César Ficelle, on voit erever. Tous ces Brocheț ne sont pas nés dans l'étang... "Is fecit eui prodest". En attendant, le consommateur ne voit que de la Carpe longue, maigre, mal dégorgée, et s'en détourne. Le marchand ne va chez le bon producteur qu'à la dernière extrémité, quand lẹ mal est fait et le cours cassé.

III. - Il est faux que la marée fasse du tort au poisson vif, comme il est faux que "la basse" reste pour compte au boucher. Ce sont des dogmes répandus par ccux qui en profitent. Les pays côtiers sont de bons clients pour l'étang, et les villes qui mangent le plus de marée sont aussi les meilleurs débouchés pour la Carpe, pour la bonne Carpe, s'entend.

C'est le revendeur qui préfère la marée : elle est morte, se découpe, se conserve (?) au frigo, n'exige pas de soins. La femme tripote sans dégout la Morue et le Colin : elle ne veut pas se mouiller les doigts ni peser " une bête qui remue et qui mord " (sic). Enfin, la marée consent une commission énorme, jusqu'à 5o \%, au revendeur.

La remarquable organisation des Casinos, magasins d'alimentation à succursales, vend à la fois de la marée et de la Carpe avec garantie syndicale, sans que l'une nuise à l'autre. Mais c'est une affaire biẹn menée. 
TV. - Partout où l'on a pu livrer aux épiciers des Carpes et des Tanches calibrées, que le client choisit lui-même dans le bac, sans pesée, le poisson vif s'est vendu. La pesée est le grand déçoût du détaillant. Si lon calibre honnêtement, si l'on vend au prix moyen du kilogramme des Carpes d'au moins 2 livres et d'au plus I. 100 grammes, sans tricherie, le public renonce à sa chère pesée.

Les cours actuels du gros incitent à sacrifier ces 25 , 50 ou même ıoo grammes supplémentaires. On n'y perdra pas en livrant par 20 ou 3 o têtes, voire par 5 têtes, aux détaillants directement : l'économie résultant de la suppression de l'intermédiaire " halles ", et du demi-grossiste, laisse une marge suffisante pour couvrir les frais de transport, et au delà.

V. - Le producteur négligent n'a aucun profit (je ne dis pas son garde ni son marchand) à pêcher pour vendre "tout venant " à 20 sous la livre. Qu'il se spécialise dans le Brochet, lequel est exclu des étangs sagement. empoissonnés en petite feuille de bonne race, car la livré de Brochet y revient à 25 louis.

Dans un étang de ro Hectares, ne mettez que votre friture invendable, et to vieilles femelles, so vieux (je ne dis pas gros...) mâles. Ajoutez avant Noël roo poignards ou Brochetons de demi-livre au plus. Vendez, avant de pêcher, vos Brochets 5 francs la livre. Ne pêchez que les Brochets. Refermez la bonde et recommencez l'an suivant. Une année sur deux, vous vendrez 600 livres de Brochet, avec des frais insignifiants.

Et laissez la Carpe à ceux qui savent la produire !

\section{L'ART D'ÉLEVER LES POISSONS D'AQUARIUM}

Par M. Marcel DAGRY

(Suite) (1)

$$
\text { I. - Poissons exotiques vivipares. }
$$

\section{Girardinus.}

Les aquariophiles désignent sous le nom de Girardinus plusieurs poissoris que les ichthyologistes répartissent entre ce genre et plusieurs autres : Gladichthys, Heterandria, Lebistes, Phalloceros, Poecillia, etc., qui, comme le Xiphopore, appartiennent à la nombreuse famille des Poeciliides.

Comnic nous entendons, sans aucune prétention scientifique, fournir seulement aux amateurs les renseignements pratiques de nature à guider

(1) Voir Bulletin : - $n^{\circ} 72$, Juin 1934, p. $328 ;-n^{\circ} 73$, Juillet, p. $18 ;-n^{\circ} 74$, Aout, p. 42. 